\title{
Spatial Correlation Features for SAR Images in a Small Sample Size Context
}

\author{
Roberto Vaccaro and Silvana Dellepiane. \\ Department of Biophysical and Electronic Engineering \\ University of Genoa - Via Opera Pia 11A - 16145 Genoa - Italy \\ Phone +39-10-3532662 Fax +39-10-3532134 \\ E-mail: roby@nina.dibe.unige.it
}

\begin{abstract}
The information related with the spatial correlation properties of textured images represents the topic of the present paper. The correlation estimate task is addressed, taking into account the stability problem when small and irregularly shaped training regions are available, as the case of Remote Sensing data of the SAR type.

In these situations, the classical estimate based on large and rectangular training areas shows a large variance and, as a consequence, classification results quality strongly decreases as the training area dimensions decrease.

The proposed approach is based on the simplified assumption of independent and separable spatial correlation properties in the slant and azimuth directions, and it takes advantage of onedimensional processing to reduce the computation load. Two one-dimensional correlation functions are then easily extracted from small and irregular training areas, and they are successively applied for a classification process, on the basis of a maximum likelihood criterion. Theoretical and experimental comparisons with the classical two-dimensional approach are presented.

Even though some information is lost in the proposed method, larger spatial neighbourhoods can be considered with only a linear increase of computation load. The results achieved on SAR test images show a significant increase in classification accuracy, proving that the simplified one-dimensional approach correctly takes into account spatial information to the end of the classification problem.
\end{abstract}

\section{Introduction}

The statistical models applied for textural feature extraction in a classification context are often too simplified, giving rise to unsatisfactory results.

For instance, in the case of SAR images, traditional classification methods, are not suitable, due to the presence of speckle noise, image complexity and limited training sets. In fact specialised models have been designed to describe the textual information in SAR images [3].

The problem of a powerful exploitation of spatial correlation properties is addressed in the present paper with the purpose of classification. In the current literature, methods exploiting such correlation properties can be found. For instance in [5] Rignot and Chellappa suggest a model for the estimate of the spatial conditional probabilities included in a broader MRF approach.

The small sample size problem affects the stability of correlation estimates, mainly as deals large correlation lags. This fact, together with the enormous computation load, is the main reason why correlation lags larger than one are not usually taken into account. 
To face these drawbacks, a method is proposed for the estimate of the spatial correlation matrix, which decomposes the bidimensional image data into monodimensional signals.

Two correlation functions are estimated in the slant and azimuth directions, respectively, on the basis of the available small training sets. A couple of $2 \times 2$ subcovariance matrixes are then constructed for each considered lag, and a modified maximum likelihood discriminant function is derived.

Some portion of the information contained in the classical spatial correlation matrix is lost but the computed coefficients show a lower variance with respect to the $2 \mathrm{D}$ case. In fact, all samples belonging to the training set are used in this case, and not only a rectangular subset of the training area.

This paper is divided in three sections. In the next session we introduce the correlation properties and we present the estimate of the correlation functions. In the third section we develop a modified maximum likelihood classifier which exploits the information of the above correlation functions. Finally, in the last section, estimate of the correlation functions and classification results as obtained on test images are presented.

\section{Estimate of the spatial correlation features for small training regions}

The spatial correlation matrix is a significant statistical property of the second order. The use of this measure in the classification task allows to take into account more accurate models and to significantly improve classification accuracy.

In general, the goodness of an estimator can be measured through two parameters: the bias and the variance. An estimator is said consistent if these two parameters tend to zero with the increasing of the observed signal.

It's possible to choose a biased or an unbiased spatial correlation estimator. The variance of the estimator is function of the size of the training area together with the leg of the spatial correlation to be estimated. Only a finite part of a signal is considered when the estimate of the spatial correlation of a signal is computed. Therefore, the size of the training area is important for the stability of the estimated spatial correlation.

The classical estimate of the spatial correlation matrix is made with the following method.

Under the hypothesis of a stationary bidimensional process, if the following neighbourhood is given:

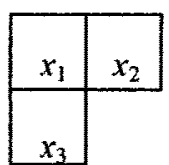

a $3 \times 3$ spatial correlation matrix can be estimated as:

$$
\rho_{2 D}=\left[\begin{array}{lll}
R_{1,1} & R_{1,2} & R_{1,3} \\
R_{1,2} & R_{1,1} & R_{2,3} \\
R_{1,3} & R_{2,3} & R_{1,1}
\end{array}\right]
$$


Such a matrix represents the bidimensional correlation values for unitary lags in horizontal, vertical, and oblique directions.

The estimator of the single element has the following shape[1] [2] [4]:

$$
R_{j k}=\frac{1}{N} \sum_{n=0}^{N-1} x_{j} x_{k}
$$

where $N$ is the number of the sites in the training area that completely contain the neighbouring mask.

As a consequence, a frame of border pixels is lost, whose dimensions depend on the mask dimension, that is, on the maximum lag.

Under these conditions, the classical estimate of the correlation matrix is stable only when it is possible to extract large and rectangular sets of neighbouring pixels from the training regions.

In the practical applications is quite usual to have training regions with irregular shape and made up of very few samples. In this case the classical way of computing the correlation matrix does not give rise to stable results.

The solution proposed in this paper consists in analysing the information carried by the bidimensional signal by means of successive analysis of azimuth and slant range directions.

Similarly to the approach followed in the classical textural estimation of run-length and cooccurrence matrices, the bidimensional signal is transformed into a monodimensional one, by putting the samples which lie in subsequent rows (or columns) in a monodimensional vector.

Such a vector is then:

\begin{tabular}{|l|l|l|l|l|l|l|}
\hline $\mathrm{x}(\mathrm{l})$ & $\cdots \cdots \cdots$ & $\mathrm{x}(\mathrm{n})$ & $\cdots$ & $\mathrm{x}(\mathrm{n}+\mathrm{j})$ & $\cdots \cdots \cdots$ & $\mathrm{x}(\mathrm{N})$ \\
\hline
\end{tabular}

and the spatial correlation information in one dimension, at lags $1,2, \ldots, \mathrm{n}$ is the following:

$$
\rho=\left[\begin{array}{llllllllll}
R_{1,1} & R_{1,2} & R_{1,3} & R_{1,4} & R_{1,5} R_{1,6} & R_{1,7} & R_{1,8} & R_{1,9} & R_{1, L+1}
\end{array}\right]
$$

The estimator of the single element has the following shape[6]:

$$
R_{1, j+1}=\frac{1}{N} \sum_{n=1}^{N-j} x(n)_{x}(n+j)
$$

$N$ is the size of the training area, $x(n)$ is a sample of the training area and $j$ is the lag of the correlation element.

In this way a stable estimate of each correlation coefficient can be achieved for $\mathrm{L}$ significant lag value. In addition, it is possible to use training windows with any shape. Finally, the number of border samples is reduced .

\section{Discriminant function}

Given the originally proposed approach for the estimation of the spatial correlation features, it is then necessary to modify the parametric structure of the maximum likelihood functions.

The novel discriminant function is derived from a sum of maximum likelihood terms computed on reduced matrices. 
The proposed idea is to generate, for each considered lag, a couple of covariances matrices, of size $2 \times 2$, composed by the coefficients estimated in the new way. Their shape is the following:

$$
\Sigma_{j}^{i}=\left[\begin{array}{cc}
R_{1,1}-\mu_{i} & R_{1, j+1}-\mu_{i} \\
R_{1, j+1}-\mu_{i} & R_{1,1}-\mu_{i}
\end{array}\right]
$$

The decision function, related to the generic class $i$, changes according to the following:

$$
\mathrm{g}_{1 D i}\left(x_{p}\right)=-\sum_{j=1}^{L} \ln \left|\Sigma_{j}\right|-\sum_{j=1}^{L}\left\{\left[\begin{array}{c}
x_{p}-\mu_{i} \\
x_{p+j}-\mu_{i}
\end{array}\right]^{t}\left(\Sigma_{j}^{i}\right)^{-1}\left[\begin{array}{c}
x_{p}-\mu_{i} \\
x_{p+j}-\mu_{i}
\end{array}\right]\right\}
$$

with $\quad p$ index of the current pixel

To compare the new discriminant function to the traditional 2D one, we consider, for simplicity, a $\Sigma_{2 D}$ of $3 \times 3$ size, for which:

This means that the oblique direction correlation is approximated with the same value as the horizontal one.

In such a case, the inverse matrix is: $\left(\Sigma^{i}\right)^{-1}=\frac{1}{\left|\Sigma^{i}\right|}\left[\begin{array}{ccc}\beta_{1} & \beta_{2} & \beta_{3} \\ \beta_{2} & \beta_{4} & \beta_{2} \\ \beta_{3} & \beta_{2} & \beta_{1}\end{array}\right]$

where: $\beta_{1}=R_{1,1}^{2}-R_{1,2}^{2} ; \beta_{2}=R_{1,2}\left(R_{1,3}-R_{1,1}\right) ; \quad \beta_{3}=R_{1,2}^{2}-R_{1,3} R_{1,1} ; \beta_{4}=R_{1,1}^{2}-R_{1,3}^{2}$

$$
\left|\Sigma^{i}\right|=R_{1,1}^{3}-2 R_{1,2}^{2} R_{1,1}+2 R_{1,2}^{2} R_{1,3}-R_{1,3}^{2} R_{1,1} \text {; }
$$

In the case of the one-dimensional approach, two correlation matrices corresponding respectively to lag 1 and 2, are: $\quad \Sigma_{1}^{-1}=\left[\begin{array}{cc}\alpha_{1} & \alpha_{2} \\ \alpha_{2} & \alpha_{1}\end{array}\right] ; \quad \Sigma_{2}^{-1}=\left[\begin{array}{ll}\alpha_{3} & \alpha_{4} \\ \alpha_{4} & \alpha_{3}\end{array}\right]$; where $\quad \alpha_{1}=\frac{R_{1,1}}{\left|\Sigma_{1}^{i}\right|} \quad \alpha_{2}=-\frac{R_{1,2}}{\left|\Sigma_{1}^{i}\right|} \quad \alpha_{3}=\frac{R_{1,1}}{\left|\Sigma_{2}^{i}\right|} \quad \alpha_{4}=-\frac{R_{1,3}}{\left|\Sigma_{2}^{i}\right|}$ with: $\quad\left|\Sigma_{1}^{i}\right|=R_{1,1}^{2}-R_{1,2}^{2}$; $\left|\Sigma_{2}^{i}\right|=R_{1,1}^{2}-R_{1,3}^{2}$;

Now, the classical ML computation give rise to:

$$
g_{2 D i}\left(x_{1}\right)=\frac{1}{\left|\Sigma^{i}\right|}\left(x_{1}^{2} \beta_{1}+x_{2}^{2} \beta_{4}+x_{3}^{2} \beta_{1}+2 x_{1} x_{2} \beta_{2}+2 x_{3} x_{2} \beta_{2}+2 x_{1} x_{3} \beta_{3}\right)+\ln \left(\left|\Sigma^{i}\right|\right)
$$

while in the proposed method, we have:

$g_{1 D i}\left(x_{1}\right)=\left(\alpha_{1}+\alpha_{3}\right) x_{1}^{2}+\left(\alpha_{1}+\alpha_{3}\right) x_{2}^{2}+2 x_{2}^{2} \alpha_{1}+2 x_{1} x_{2} \alpha_{2}+2 x_{2} x_{3} \alpha_{2}+2 x_{1} x_{3} \alpha_{4}+\ln \left(\left|\Sigma_{1}^{t}\right| \cdot \mid \Sigma_{2}^{t}\right)$

While in the 2D approach, the computation of the inverse matrix has an exponential growth with the increase of the lag value, with the new method, the exploitation of lag up to 2 does not cause an intractable increase of complexity. In fact adding a new lag only cause a linear increase of computation.

As already noticed, if the two methods are applied on the same training region, the coefficients of the correlation estimates in the second way are more stable than the classical ones.

As a consequence, a portion of the lost $2 \mathrm{D}$ information is recovered trough the exploitation of the additional correlation coefficients. 
Finally, in a small sample size context, the proposed method becomes particularly interesting.

\section{Results}

We have divided the results into two sections. In the first part we present the estimates of the correlation functions as computed on SAR images. The behaviour of the functions and the variance of the estimated values are presented and discussed through the presentation of some graphics.

In the second part some classification results obtained with the modified discrimination function are reported. The classification is made on the basis of test images that contain objects with irregular shape.

\subsection{Estimate of the correlation function on real SAR data}

We have estimated the correlation function in the azimuth and in the slant range direction on a few SAR images.

The images used for this test belong to the Maestro 1 campaign (fig. 4.1) [7].

The behaviour of the correlation functions of the five classes in the azimuth direction is shown in Fig. 1 for lag up to 10.

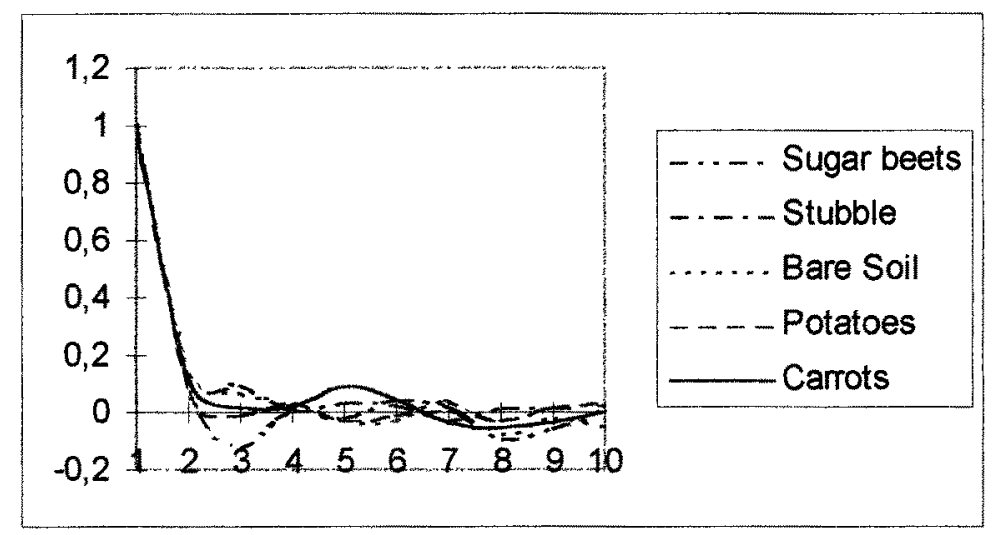

Fig. 1 Correlation functions for the five classes present in the Feltwell site image.

One can observe that the value of the correlation function, even though small, is not zero also for a lag of six or nine samples, and varies for each class, thus representing a possible discriminant feature.

Moreover, the behaviour of the correlation function confirms the results theorised by Rignot and Ulaby $[3],[5]$. In fact, in their works they proved that the spatial correlation has a shape similar to a sinc or to a sinc ${ }^{2}$, which is not so different from the present results.

The training areas used for the estimate of the correlation function have been taken with different sizes as reported in Table 1 .

\begin{tabular}{|lccccc|}
\hline & Sugar beets & Stubble & Baif soil & Potatoes & Carrots \\
\hline Number of samples: & 682 & 513 & 462 & 1222 & 525 \\
\hline
\end{tabular}

Tab. I Size of the training areas used for the estimate of the correlation function. 
The variance of an estimated correlation function depends on the size of the training area as well as on the absolute value of the estimated correlation function itself. The behaviour of such variances can be seen in Fig. 2 .

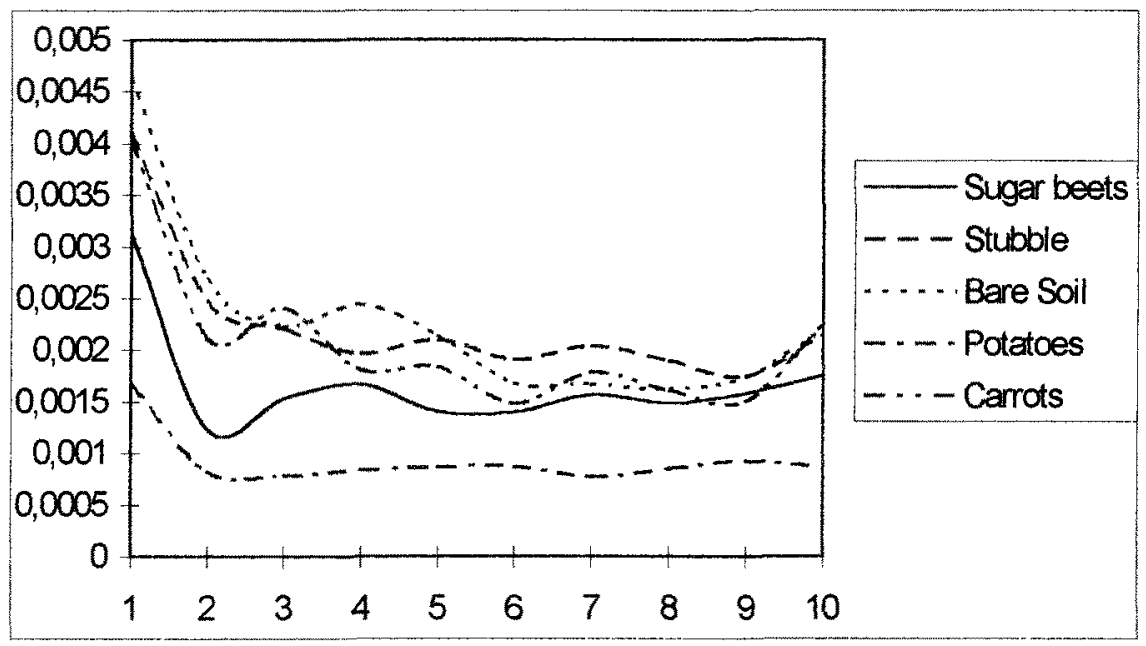

Fig. 2 Variance of the correlation functions of Fig 2.

One can observe that the variance is low when the training area is large but it increases when the size is because of the increased lag value.

\subsection{Comparison of classification results.}

We have carried out some classification trials on different kinds of images to test the theoretical models explained in the previous sections.

At first we have considered three test images generated from SAR data presenting two different classes (Fig.3). The first image has a very large background (second class) and a thin object class (first class) with a regular shape. On the contrary, the second and the third images have the second object class with an irregular shape.
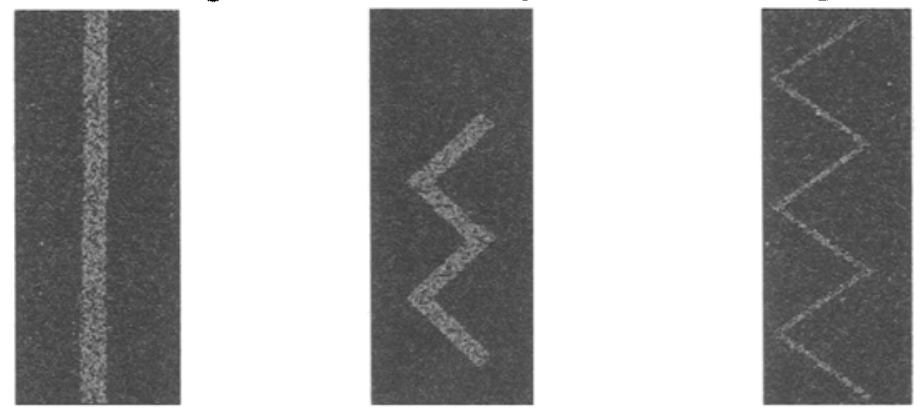

Fig. 3 Test images used for the classification tests.

We have used large training sets on the first image (over 1500 samples), and a classification process was computed with the classical and the proposed method. In this case, we have obtained better results with the classical maximum likelihood as can be seen in Table. 2 . 


\begin{tabular}{|crr|}
\hline & Classical & Proposed \\
\hline Class 1 & 91,76 & 86,59 \\
\hline Class 2 & 98,25 & 94,62 \\
\hline
\end{tabular}

Tab. 2 Classification accuracy achieved

on the first image for the two methods.

This behaviour is due to the information loss of the proposed method and by the size of the training area which is large enough also for the classical approach. In this case, in fact, the advantages of the proposed methods are not significant as the training set is not small or irregular.

On the contrary, we have used a small training area (16 samples) to estimate the features of the irregular first class, in the second image. The results can be seen in Table 3.

Now the results we have obtained are better for the proposed method because the correlation matrix estimated with the classical method is not stable enough.

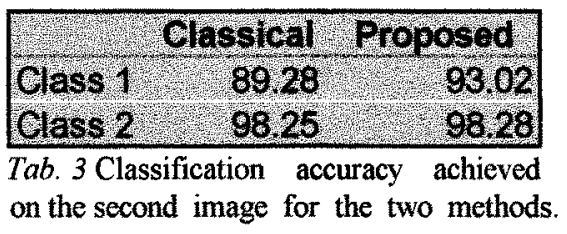

It is easy to notice that the monodimensional method works better than the classical one if the goal is the recognition of patterns which do not allow the classical method to have large regular training areas. This difference of quality is more evident in the pixels near the border. If we compute the accuracy in the areas close to the border of class 1 (red colour) we find (Table 4) in those places, the accuracy achieved with the classical method is very poor:

\begin{tabular}{|c|cc|}
\hline & Classical & Proposed \\
\hline Class 1 & 3,46 & 90,52 \\
\hline Tab. 4 Classification accuracy for the \\
Two methods close to the border.
\end{tabular}

This result is due to the fact that in the new method we have computed ten elements of the correlation.

Finally the results obtained from the third image can be seen in Fig. 4.1 and in Fig. 4.2. We can observe (Table 5) that the accuracies are better for the modified classifier.

\begin{tabular}{|ccr|}
\hline & Classical & Proposed \\
\hline Class1 & 57,85 & 94,05 \\
\hline Class2 & 96,88 & 88.62 \\
\hline
\end{tabular}

Tab. 5 Classification accuracy achieved on the second image for the two methods.

On the contrary, the classical method achieves better results for the background object because is available a large and regular region. In conclusion the new proposed method is more robust in border areas. 


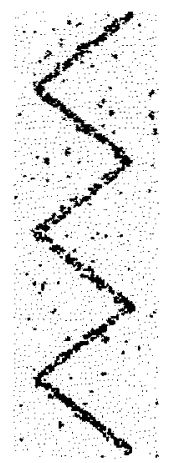

Fig. 4.1 Classification map of the third image as obtained by the classical method.

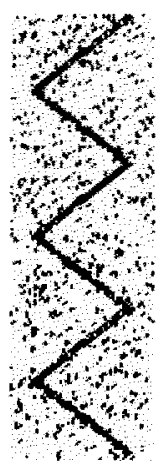

Fig. 4.2 Classification map of the third image as obtained by the proposed method.

\section{Conclusions}

The experimental data confirm that the spatial correlation is an important statistical property.

By exploiting this information during features extraction it is possible to achieve an improvement in the accuracy quality of the classification results because the models are more complete.

Finally, the method for the monodimensional estimate of the correlation and the method for the modified computation of the maximum likelihood are effective in those situations where the recognition of thin and not regular shaped objects does not allow the classical estimate methods to achieve satisfactory results because of the instability of the estimates.

\section{References}

[1] Athanasios Papoulis, "Probability, Random Variables, and Stochastic Processes", McGraw-Hill International Editions, 1987.

[2] A.Bruce Carlson, "Comunication Systems", McGraw-Hill International Editions, 1987.

[3] F.T.Ulaby, F.Kouyate, B. Brisco, T.H.Lee Williams "Textural information in SAR Images", IEE transaction on Geoscience and Remote Sensing, Vol. GE-24, No 2 March 1986

[4] J.P Hoffbeck,D.A. Landgrebe, "Covariance Estimation for Classifing High Dimensional Data", 0-7803-2567-2,IEEE, 1995.

[5]E. Rignot, R. Chellappa,"Segmentation of Synthetic-aperture -radar complex data", J.Opt. Soc.Am. A/Vol, 8, No. 9/September 1991.

[6] A.V.Oppenheim,R.W. Shafer,"Elaborazione numerica dei segnali", Franco Angeli, 1990.

[7] MAESTRO 1, Airborne Synthetic Aperture Radar (SAR) Campaign, "DATA REPORT", European Space Agency, 1990. 